\title{
RUPTURA UFMG: EVENTO DE INOVA ÇÃ O E EMPREENDEDORISMO DA ESCOLA DE ENGENHARIA DA UFMG
}

DOI: 10.37702/2175-957X.COBENGE.2021.3588

Alessandro Fernandes Moreira - moreira@cpdee.ufmg.br Universidade Federal de Minas Gerais

Rua Dom Prudêncio Gomes 168

30535-580 - Belo Horizonte - MG

Karine Fernandes Brandão - karinebrandao8@gmail.com Universidade Federal de Minas Gerais

Av. Pres. Antônio Carlos 6627

31270-901 - Belo Horizonte - MG

Ana Paula Pereira Theobald - anaptheobald@gmail.com

Universidade Federal de Minas Gerais

Rua Martinica 432

31565-400 - Belo Horizonte - MG

Hanna Rodrigues - rdg.hanna@gmail.com

Universidade Federal de Minas Gerais

Rua Ilha Grande 300

31555-030 - BELO HORIZONTE - MG

Maria Luiza Coelho Rodrigues - malucrodriguess1@gmail.com Universidade Federal de Minas Gerais

Rua Mércia Siqueira Prates 39

31550-370 - Belo Horizonte - MG

Karenina Favoreto Pessi - karenina.favoreto@hotmail.com Universidade Federal de Minas Gerais

Rua Noraldino de Lima 290

31270-650 - Belo Horizonte - MG

Joanna Pinheiro Rodrigues - joannaprodrigues@gmail.com Universidade Federal de Minas Gerais

Rua João Gualberto Filho 1260

31035-570 - Belo Horizonte - MG 
Magno Côrrea de Morais Costa - magnocmc3@gmail.com

Universidade Federal de Minas Gerais

Rua Papoula Vermelha 245

30555-190 - Belo Horizonte - MG

Rayssa Sarah de Oliveira Guimarães - Rayssasog@gmail.com

Universidade Federal de Minas Gerais

Rua presidente Nilo Peçanha 440

33035-240 - Santa Luzia - MG

Resumo: Este trabalho apresenta o Ruptura UFMG, evento de empreendedorismo e inovação realizado pelo Programa ENG200 da Escola de Engenharia da UFMG em parceria com o IEBT e a TRIE Engenharia. Inspirado no evento EPICENTRO e desenvolvido aos moldes do Bootcamp de Empreendedorismo do SCET/UC-Berkeley, o Ruptura UFMG tem como ponto forte o protagonismo estudantil. Apoiado por empresas do ecossistema de inovação $e$ empreendedorismo de Belo Horizonte, o Ruptura UFMG possui uma natureza disruptiva, de forma que o evento se reinventa a cada edição, seja realizado de forma presencial ou online, promovendo uma sinergia entre o mercado de trabalho e o meio universitário e proporcionado um ambiente favorável para troca de experiências. Totalmente alinhado com as novas Diretrizes Nacionais Curriculares (DCNs) das Engenharias, o Ruptura UFMG é uma atividade acadêmica complementar em formato de evento que agrega competências necessárias à formação do Engenheiro neste mundo contemporâneo e globalizado.

Palavras-chave: Inovação e Empreendedorismo; Atividades Acadêmicas Complementares; Eventos de Educação; Inovação na Educação em Engenharia. 


\section{RUPTURA UFMG: EVENTO DE INOVAÇÃO E EMPREENDEDORISMO DA ESCOLA DE ENGENHARIA DA UFMG}

\section{INTRODUÇÃO}

Nos últimos anos, "Engenheiro do Futuro" tem sido um tema recorrente em fóruns de discussão de Educação em Engenharia e, consequentemente, as habilidades que esse profissional precisa desenvolver nas instituições de ensino superior para atender as necessidades e expectativas do mercado de trabalho (AGRELA, 2018).

O desenvolvimento exponencial de novas tecnologias, as novas demandas da sociedade e a pandemia Coronavirus Disease 2019 (Covid-19), do ano de 2020, trouxeram grandes impactos à sociedade, tendo por consequência o inevitável dever de se adaptar as profissões e os profissionais a essa nova realidade. Isso se torna evidente, sobretudo, quando se trata do profissional de engenharia, pois essa é a área que responde em grande parte pelo desenvolvimento tecnológico da sociedade, sendo imprescindível a constante evolução de suas habilidades, de acordo com o contexto social e técnico em que se vive (PAIVA; ASSAYAG; GARCEZ, 2019). Frente a isso, as Instituições de Ensino Superior (IES) precisam apresentar novas formas de desenvolver tais habilidades, além de promover uma correlação mais efetiva entre o meio acadêmico e o mercado de trabalho, que é mais adaptável às mudanças, e já se encontra imerso nessa nova sociedade e carente de profissionais capazes de lidar com ela.

Neste contexto, as mudanças no processo de ensino-aprendizagem nas faculdades de engenharia, bem como os projetos e práticas pedagógicas, têm se tornado pauta recorrente, em prol de aproximá-los cada vez mais do mercado de trabalho, em conformidade com a nova resolução de Diretrizes Curriculares Nacionais do Curso de Graduação em Engenharia (Resolução CNE/CSE 02/2019), publicada em abril de 2019, em que, especialmente no Capítulo III - Da Organização do Curso de Graduação em Engenharia, art. $6^{\circ}$, inciso 9으. apresenta:

"É recomendável que as atividades sejam organizadas de modo que aproxime os estudantes do ambiente profissional, criando formas de interação entre a instituição e o campo de atuação dos egressos."

Frente a estes desafios, uma das estratégias para a promoção de ambientes acadêmicos inovadores e propícios ao desenvolvimento de novas habilidades é o fomento da cultura do empreendedorismo. A palavra "empreendedorismo", comumente associada à criação de novos negócios, vem ganhando cada vez mais espaço nas IES de Engenharia. Nesse sentido, é importante destacar que "empreender" vai além de simplesmente criar empresas (SILVA et al., 2015): é uma mentalidade que carrega consigo características imprescindíveis para os engenheiros que o mercado necessita, como autonomia, liderança, cooperativismo, criatividade, gestão de pessoas, entre outras (BUENO, 2019). Além disso, as atividades acadêmicas complementares também são de extrema importância nesse contexto. Complementares às atividades obrigatórias e optativas, elas são mais adaptáveis a renovações estruturais para responderem às novas demandas. Nesse sentido, eventos acadêmicos e científicos, promovidos pelas IES, são atividades que agregam as duas estratégias supracitadas em prol de criar "mecanismos e instrumentos úteis para 0 despertar de um 'espírito' empreendedor e de inovação nos seus estudantes” (SILVA et al., 2015).

Dessa forma, com a intenção de qualificar egressos de Engenharia "para que estejam aptos a atuar no mercado profissional com o que há de mais novo em métodos e 
processos tecnológicos, propiciando a formação de profissionais, com caráter responsivo a estas mudanças" (PAIVA; ASSAYAG; GARCEZ, 2019), a Escola de Engenharia da UFMG (EEUFMG), desde de 2016, promove anualmente o evento "Ruptura UFMG", em parceria com diversas empresas e instituições.

Este trabalho tem como objetivo demonstrar que a realização de eventos acadêmicos e científicos de inovação e empreendedorismo constituem um papel fundamental na formação do chamado "engenheiro do futuro", e seguem as expectativas do desenvolvimento do perfil e competências esperados dos novos profissionais, sobretudo porque estes eventos podem ser realizados a partir de parcerias com empresas e indústrias. São apresentadas a concepção e as edições do Ruptura UFMG, dando foco à sua adaptabilidade e aperfeiçoamento, bem como seu impacto na formação de profissionais para o futuro e sua relação com o mercado de trabalho.

\section{A ESTRUTURA DO EVENTO RUPTURA UFMG}

O Ruptura UFMG foi criado em 2016, inspirado no EPICENTRO, evento nacional sobre empreendedorismo e criatividade que acontece em Campos do Jordão, São Paulo (https://www.oepicentro.com.br/). A principal missão do Ruptura UFMG é desconstruir a ideia do empreendedorismo apenas como o ato de abrir sua própria empresa, mostrando que empreender é também mobilizar recursos diversos para realizar projetos que tenham um impacto positivo na sociedade (BUENO, 2019) e incentivar o protagonismo estudantil. Desse modo, o seu nome carrega o propósito do evento, que é ser disruptivo, a partir do rompimento com o senso comum e a promoção da inovação para os seus participantes.

Desde sua primeira edição, o Ruptura UFMG tem apoio de empresas atuantes no ecossistema de empreendedorismo de Belo Horizonte para sua realização. Organizado pelo Programa de Inovação em Educação em Engenharia - Programa ENG200 (MOREIRA et al., 2013) em conjunto com a empresa IEBT, teve a participação inicial da empresa Optimus Engenharia. Atualmente, realizam o evento, além do Programa ENG200 e do IEBT, a empresa TRIE Engenharia. Na sua sexta edição, o Ruptura UFMG se consolida a cada ano e se adapta às novas tendências de mercado para poder sempre ser de grande valia para os seus participantes.

O evento é apoiado por empresas parceiras, que fornecem o aporte financeiro necessário para a sua realização e, em algumas ocasiões, conteúdo técnico para o evento. Entende-se que a participação dessas empresas também contribui para o Ruptura UFMG se tornar mais atrativo para os estudantes, uma vez que muitos participantes veem oportunidades no contato direto que tem com os profissionais das empresas. Além disso, algumas empresas também oferecem premiações para as competições que o evento tem em seu conteúdo, que variam desde bens materiais até vagas de estágio. Dentre as empresas que participaram do Ruptura UFMG, podem ser citadas a FCA (Fiat Chrysler Automobiles), que teve grande participação no conteúdo da segunda edição, a EMBRAER, o Banco Santander, VLI Logística, Belgo Bekaert Arames e a Voitto.

Em 2020, na 5ำ edição do Ruptura UFMG, a Wabtec Corporation e o IEBT foram corealizadoras do evento, sendo empresas essenciais para a estruturação dos desafios propostos. No que concerne aos objetivos do evento, o Ruptura UFMG entrega muito mais que apenas soluções para as temáticas e problemas propostos nos desafios. Durante o evento, os gestores e funcionários das empresas parceiras ficam imersos juntamente aos participantes em um ambiente de inovação, o que influencia na mudança de mentalidade interna da própria empresa. A presença no ambiente universitário, inovador e disruptivo, fonte de talentos e oportunidades para captação de colaboradores, é de grande importância para as empresas participantes. Em contrapartida, para a IES, a presença das empresas 
na formação dos estudantes agrega valores importantes no perfil de formação dos/as futuros/as engenheiros/as. Um dos fatores essenciais para o sucesso do evento é a sua forma de organização, que sempre contou com a participação efetiva dos estudantes, não apenas para sua execução, mas, também, na concepção da temática e dos conteúdos. $O$ Ruptura UFMG é feito para estudantes e pelos estudantes. Essa característica é de grande importância, uma vez que a cada edição consegue-se captar quais as necessidades dos estudantes e qual a "ruptura" que precisa ser realizada.

A cada edição, o evento Ruptura UFMG se renova e modifica seu formato, conteúdo e estrutura de organização, tornando o evento sempre atual. Quando se fala de inovação, no contexto atual, as mudanças ocorrem de maneira muito rápida e muitas vezes não se consegue trabalhar no ensino formal, com a rapidez com que são exigidas, as competências necessárias para lidar com tais mudanças. A essência mutável do Ruptura UFMG o torna sempre vanguardista, inovador e atrativo. Sem a imposição de seguir um modelo de evento, cada edição se torna disruptiva por si só, criando uma experiência totalmente atual e voltada para o profissional do futuro. Por conseguinte, a adaptação do evento para o formato online, devido à pandemia de Covid-19 em 2020, não foi um grande desafio, mas sim natural à essência do evento.

\section{A HISTÓRIA DO RUPTURA UFMG}

A primeira edição, em 2016, teve como objetivo principal conscientizar todo universitário sobre seu potencial transformador, como um agente capaz de identificar e resolver os problemas da sociedade. Objetivou-se, também, instigar toda a comunidade acadêmica a ter um comportamento mais proativo, inovador, social e generoso. Adicionalmente, pretendeu-se aproximar a comunidade acadêmica da UFMG ao ecossistema de startups, para que os alunos pudessem ver o empreendedorismo de uma forma mais cotidiana e palpável. Nesse sentido, a partir da exposição por parte de empresas de seus produtos e ideias, foi possível imergir os estudantes no pólo de inovação e empreendedorismo presentes em Belo Horizonte. Um dos pontos principais desta edição foi a participação do Sutardja Center for Entrepreneurship and Technology (S/CET) da University of California-Berkeley (https://scet.berkeley.edu/). Os profissionais do S/CET desenvolveram, junto com equipe do Ruptura UFMG, um bootcamp, que consistiu em uma competição na qual os participantes do evento, divididos em grupos de 4 ou 5 pessoas, realizavam as mais diversas atividades em busca de adquirirem noções sobre empreendedorismo e sobre como validar novas ideias e desenvolvê-las até chegarem em uma proposta de produto ou serviço. Com a participação de mais de 200 pessoas, o evento pôde ser ampliado e repensado para que fosse ainda mais impactante no ano seguinte.

Em 2017, o Ruptura UFMG propôs o tema "Empreendedorismo e Eupreendedorismo" e organizou palestras com temas focados em habilidades pessoais em cada participante tais como liderança, oratória e empatia. Adicionalmente, propôs a discussão de metodologias para gerenciamento de projetos, estratégias de marketing e modelagem financeira, temas estes muito importantes para despertar o ser empreendedor nos estudantes. Para alcançar a temática do "Empreendedorismo e do Eupreendedorismo" foram propostos dois caminhos de palestras que os participantes: um focado mais no desenvolvimento pessoal, e outro no desenvolvimento empresarial. Contando com quatro dias de conteúdo, o evento teve a participação de grandes empresas, como a FCA, EMBRAER e o Banco Inter, que contribuíram tanto financeiramente como agregaram conteúdo a todas as discussões.

O Ruptura UFMG 3.0 continuou a inovar e teve como tema o Empreendedorismo para o Desenvolvimento Social, assunto relevante no ano de 2018 e que continua em 
crescimento. Naquele ano, o Ruptura UFMG teve seu conteúdo focado em novos nichos de mercado, em oficinas multidisciplinares e na resolução de desafios durante todo o evento. Além disso, aconteceu uma imersão noturna, na qual os participantes foram instigados a resolver, durante a madrugada, desafios propostos por empresas como a Belgo Bekaert Arames, o Banco Santander, além de algumas ONGs e associações, como O Litro de Luz.

Em 2019, o Ruptura UFMG foi reestruturado para um formato de hackathon, o que se mostrou um sucesso. Realizado em um número menor de dias, porém extremamente imersivo, com o tema "Ruptura UFMG 4.0", teve o propósito de se discutir e pensar em soluções que envolviam a quarta revolução industrial, também chamada de indústria 4.0 (PERASSO, 2016). O Ruptura UFMG 4.0 teve como temática a resolução de problemas reais de empresas destaques no mercado, Belgo Bekaert Arames e VLI Logística, as quais apresentaram problemas específicos em forma de desafios para os participantes elaborarem soluções inovadoras. Com o objetivo de preparar os participantes, foi realizado um dia de mentorias, guiadas por profissionais das duas empresas e especialistas convidados a fim de fomentar o protagonismo estudantil e evidenciar o seu potencial transformador no contato com o mercado. O evento também incluiu uma imersão noturna, que ocorreu no Parque Tecnológico de Belo Horizonte (BH-TEC).

O ano de 2020 foi marcado por expressivas mudanças nos âmbitos ambientais, geopolíticos, econômicos e cotidianos, devido à pandemia de Covid-19. O impacto nas IES, em decorrência da suspensão das atividades acadêmicas presenciais, fez com que os esforços de inovação nas instituições fossem voltados para adaptação ao sistema remoto de ensino, ampliando o distanciamento entre a academia e o mercado de trabalho. Ademais, o impacto econômico da pandemia nas empresas, repercutiu diretamente nos setores de inovação: o "mercado de inovação estima a perda aproximada de US\$1 bilhão pelo cancelamento de eventos, estes importantes espaços de negociação" (CIRNE, 2020), reforçando a necessidade e continuidade, mesmo que de forma remota, de eventos como o Ruptura UFMG.

Tendo em vista o sucesso do formato de hackathon no ano anterior, a edição de 2020 manteve este formato. Contudo, a estrutura do evento teve que ser totalmente repensada, de maneira a possibilitar que ele ocorresse de forma integralmente remota. Em virtude da necessidade de adaptação que o contexto em questão trouxe para todas as

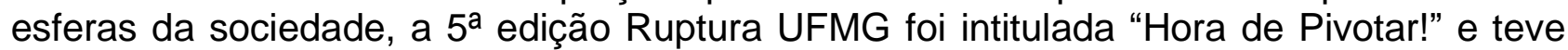
como tema a "mudança". O objetivo do tema proposto foi instigar os participantes a analisarem os prós e contras das atuais condutas da sociedade, de forma a promover mudanças benéficas e duradouras, tendo em vista que, devido à pandemia de Covid-19, vários aspectos da vida cotidiana tiveram que ser repensados, tanto na esfera pessoal quanto profissional, forçando uma adaptação em novas rotinas, ambientes de trabalho, hábitos e formas de se relacionar e de se comunicar. Essa última edição do Ruptura UFMG será abordada em mais detalhes na próxima sessão.

\section{A 5 EDIÇÃO DO RUPTURA UFMG: HORA DE PIVOTAR!}

A 5a edição do Ruptura UFMG ocorreu entre os dias 24 e 27 de Setembro de 2020 e teve como referência a edição anterior do evento, seguindo os moldes de um hackathon, mas pela primeira vez no formato remoto. O termo hackathon se refere a um evento onde programadores de diversas áreas se reúnem para criar uma solução para um problema específico e competem entre si por meio da defesa de suas ideias (BRISCOE; MULLIGAN, 2014). Entretanto, a estrutura do evento foi adaptada para que o foco principal fosse a resolução de um problema por uma equipe multidisciplinar, sem a dependência de 
desenvolver estratégias de programação. Ademais, houve a incorporação de palestras, dando enfoque na transmissão de conteúdos práticos para os participantes, além dos desafios, com o intuito de priorizar conhecimentos que poderiam ser úteis não somente para resolução do desafio, mas também para o desenvolvimento pessoal e profissional dos participantes. Por fim, a edição contou com discussões acerca do atual momento econômico e social do Brasil e do mundo, pautando-se nos pilares da inovação e das relações interpessoais.

O Ruptura UFMG - Hora de Pivotar! foi realizado de forma remota, a partir da integração de quatro plataformas online: Discord, Microsoft Teams, Ideaboardz e Shawee. Um servidor do Discord, "plataforma de comunicação instantânea que permite a troca de mensagens em texto, áudio e vídeo" (Magalhães, 2020), foi utilizado como plataforma principal do evento para centralização de informações, divisão dos grupos e mentorias, além de contar com salas de descontração e integração. O Microsoft Teams foi utilizado como plataforma de vídeo-conferências para as palestras, cujos comentários e perguntas eram feitos por meio do Ideaboardz, uma ferramenta de quadro branco virtual. A plataforma da Shawee, empresa parceira especializada em Hackathons online, foi usada para o cadastramento de todos os participantes do desafio, assim como dos mentores e jurados da banca final.

O evento contou com a participação da Wabtec Corporation e do IEBT como empresas co-realizadoras. Além destas, a TRIE Engenharia e a ISVOR também foram patrocinadoras, assim como a Voitto, a Fundação Christiano Ottoni e a própria EEUFMG também.

A temática da edição foi estruturada com a participação das empresas Wabtec Corporation e do IEBT, as quais apresentaram problemas específicos em forma de desafios para os participantes elaborarem soluções inovadoras. Os desafios, foram, respectivamente, "Sustentabilidade no Mercado Ferroviário" e "Transformação Digital e data analytics". Com o objetivo de capacitar os participantes para atuarem nos cases, uma série de materiais sobre resolução de problemas foi disponibilizado e ao longo da programação do evento ocorreram diversas mentorias exclusivas para cada grupo, guiadas por cerca de quarenta (40) profissionais das duas empresas e especialistas convidados. Nessas mentorias, temas estruturantes foram abordados tais como perspectivas de mercado, modelagem de negócios, modelagem financeira e pitch. Os participantes foram divididos em equipes, que trabalharam durante todo o evento para desenvolverem soluções para os problemas propostos pelas empresas. No último dia de programação, os grupos enviaram um sumário executivo de até duas páginas, explicando as soluções propostas. Ao final, vinte e um (21) sumários foram analisados e as melhores soluções foram classificadas para uma banca de seleção final, formada pela equipe organizadora do Ruptura UFMG, mentores e pelos responsáveis de inovação das empresas parceiras.

Ambas as empresas ofereceram prêmios às melhores soluções apresentadas, com anúncio feito durante a cerimônia de encerramento do evento. Os grupos que alcançaram o primeiro lugar, em seus respectivos desafios, foram premiados da seguinte forma:

- IEBT: Cada integrante do grupo ganhou um dispositivo Kindle, um fone de ouvido e a entrada em uma etapa avançada do processo seletivo de estágio da empresa;

- Wabtec: Cada integrante do grupo ganhou um kit homeoffice (com teclado, suporte para notebook e mouse), um curso Innovation Room da Weme School, além de R\$1.000,00, que foram divididos igualmente entre os integrantes.

A programação contou ainda com as palestras "Pivotagens que Inspiram", com convidados expondo histórias e experiências de transformações e mudanças, nos âmbitos pessoais e profissionais. O principal ponto de atenção da equipe organizadora do Ruptura UFMG, para a edição online, foi preservar a essência do evento, criando uma experiência 
marcante e mantendo o engajamento dos participantes, mesmo que de forma remota. Para alcançar esse objetivo, foi enviado para cada participante um kit do evento (Figura 1), e ao longo da programação, ocorreram quizzes com o intuito de engajar mais os participantes por meio de estratégias de gamificação, com perguntas sobre todas as edições do Ruptura, sobre as empresas IEBT e Wabtec Corporation, bem como os desafios propostos por elas.

Figura 1 - Kit do participante da edição de 2020 do Ruptura UFMG, composto por uma cartela de adesivos, uma máscara, um copo, um bloco de post-its, uma caneta, um mouse pad e dois Red Bulls.

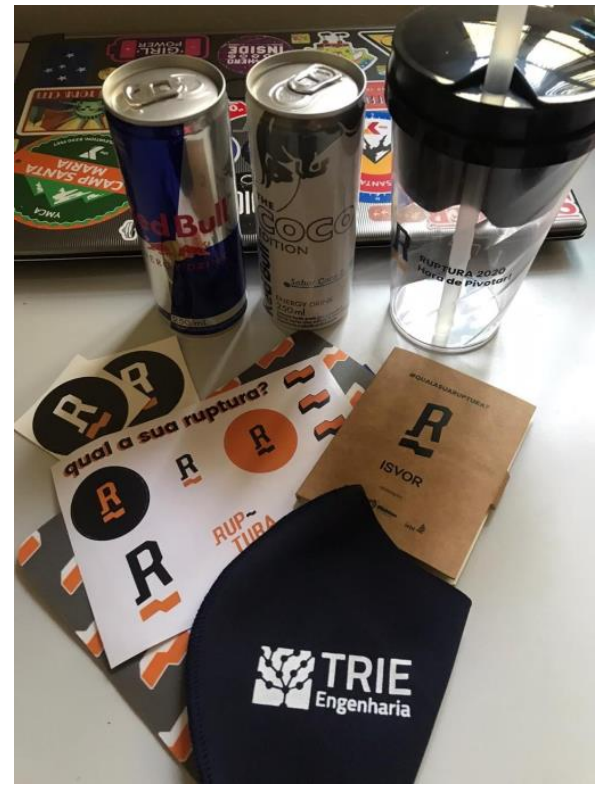

Fonte: Organização Ruptura UFMG (Programa ENG200).

O Ruptura UFMG - Hora de Pivotar! contou com cento e setenta (170) inscritos de nove (9) estados do país, sendo eles alguns funcionários das empresas parceiras e alunos de graduação e de pós-graduação de vinte e cinco (25) cursos diferentes. Em vista das edições anteriores teve um custo menor de realização, com orçamento total de cerca de $\mathrm{R} \$ 8.000,00$.

O evento foi considerado um sucesso, tanto pelos alunos envolvidos quanto pelas empresas parceiras. O formato de hackathon online, com mentorias e palestras, teve bom resultado por promover uma alta interação do participante com as empresas e entre si, bem como a manutenção da experiência do evento para o formato remoto e grande troca de vivências. Vários depoimentos foram realizados pelos participantes do Ruptura UFMG e alguns deles são apresentados a seguir, atestando a relevância do evento:

"A pouco tempo tive uma das melhores viradas de chave da minha vida. $O$ Ruptura me desafiou do início ao fim, com grandes surpresas e aprendizados de uma forma totalmente diferenciada. Sai da caixinha e construí, junto ao meu grupo incrível, um projeto que alinhava o que a empresa queria e os meus valores. Hoje colho frutos de uma dedicação e da aplicação do meu amor pela Engenharia. À toda organização, empresas e participantes, ano que vem estou aí novamente. Muito obrigado." (Participante do desafio Wabtec do Ruptura UFMG de 2020).

"Participar do Ruptura foi uma experiência tanto engrandecedora quanto desafiadora. No Direito muitas vezes não vemos o quanto a tecnologia e as 
mudanças, além da interdisciplinaridade, são essenciais à formação do bom profissional, e o Ruptura muda isso por completo. Nesses dias, trabalhei com pessoas da saúde e da engenharia, lidamos com cases reais de empresas e planilhas, tudo muito fora da minha realidade habitual, mas cheio de crescimento e aprendizado, desde a plataforma escolhida quanto os desafios e a disponibilidade da equipe, tudo contribuiu para um excelente evento, mesmo que à distância." (Participante do IEBT Ruptura UFMG de 2020).

"Esta foi a terceira edição do Ruptura que tive o prazer de organizar e executar representando o IEBT. Em cada uma delas os desafios foram diferentes, mas com experiências sempre impactantes! [...] O evento foi incrível, repleto de palestras, conteúdos, mentorias e momentos de descontração que me fizeram ter um orgulho imenso de fazer parte disso mais uma vez! [...] A toda equipe do ENG200, palestrantes, mentores e participantes minha gratidão por terem feito desse evento um dos mais marcantes na minha vida!" (Franciele Garcia, IEBT, parceira na organização do Ruptura UFMG).

\section{HABILIDADES DESENVOLVIDAS NO RUPTURA UFMG}

O Ruptura UFMG é um evento pensado para desenvolver as mais diversas habilidades, sejam elas pessoais ou profissionais, de seus participantes. Por meio de diversas palestras e treinamentos, quem tem a oportunidade de participar do evento desenvolve novas habilidades, como gerenciamento de projetos, aprender novas metodologias de gestão e além de conteúdos específicos ministrados nos workshops.

Um grande exemplo de habilidades específicas desenvolvidas no Ruptura UFMG foi, em 2018, um treinamento destinado a ensinar aos participantes estratégias para mapear e entender melhor o público alvo de seu negócio. Desse modo, os participantes foram expostos a métodos e meios de pesquisa de público, estratégias para interpretar o cliente e como vender o produto da sua empresa. Outras habilidades como oratória, trabalho em equipe e organização também foram desenvolvidas e praticadas durante o evento.

As últimas edições agregaram um caráter competitivo ao evento, já que os participantes deveriam exibir suas soluções a uma banca avaliadora. A fim de atingir esse objetivo, eles receberam treinamentos de oratória, marketing, design de apresentações e de como fazer um pitch.

Os depoimentos supracitados demonstram a importância de eventos como o Ruptura UFMG para a formação profissional do estudante, que se propõem a discutir temas variados e buscam incentivar habilidades que são ensinadas no curso de Graduação. A Tabela 1 correlaciona as temáticas nos vários eventos Ruptura UFMG e as habilidades que foram desenvolvidas. 
Tabela 1 - Habilidades Desenvolvidas nas edições do Ruptura UFMG

\begin{tabular}{c|c|c}
\hline Temática & Ano de realização & Habilidades desenvolvidas \\
\hline $\begin{array}{c}\text { Empreendedorismo } \\
\text { na prática }\end{array}$ & 2016 & $\begin{array}{c}\text { Liderança; trabalho em equipe; olhar } \\
\text { inovador; validação de ideia. }\end{array}$ \\
\hline $\begin{array}{c}\text { Empreendedorismo e } \\
\text { Eupreendedorismo }\end{array}$ & 2017 & $\begin{array}{c}\text { Oratória; trabalho em equipe; liderança; } \\
\text { modelagem financeira; gerenciamento de } \\
\text { projetos. }\end{array}$ \\
\hline $\begin{array}{c}\text { Empreendedorismo } \\
\text { Social }\end{array}$ & 2018 & $\begin{array}{c}\text { Empatia; trabalho em } \\
\text { equipe; liderança; gerenciamento de } \\
\text { tempo e de projetos; estratégias de venda. }\end{array}$ \\
\hline $\begin{array}{c}\text { Indústria 4.0 } \\
\text { Hora de Pivotar }\end{array}$ & 2019 & $\begin{array}{c}\text { Trabalho em equipe; oratória; produção de } \\
\text { conteúdo; liderança; criatividade; design } \\
\text { de apresentações. }\end{array}$ \\
\hline
\end{tabular}

Fonte: Organização Engenharia Ruptura UFMG (Programa ENG200)

\section{CONSIDERAÇÕES FINAIS}

Este trabalho apresentou o evento Ruptura UFMG, uma atividade acadêmica complementar em formato de evento de inovação e empreendedorismo que é organizado por alunos de Engenharia e realizado pelo Programa ENG200 em parceria com o IEBT e com a TRIE Engenharia. Este evento busca o desenvolvimento de habilidades e competências necessárias aos egressos de Engenharia para os novos desafios do atual cenário tecnológico e social do mercado de trabalho.

O Ruptura UFMG desenvolve competências em alinhamento total com as novas DCNs de Engenharia e tem no protagonismo estudantil um dos seus pontos mais fortes. Trabalho em equipe, oratória, liderança, criatividade, design e venda de produtos, estratégias de marketing, dentre outras, são habilidades desenvolvidas nos eventos Ruptura UFMG. Tendo como princípio o protagonismo estudantil e o compromisso em ser disruptivo e mutável a cada edição, o Ruptura UFMG contribui para a formação dos estudantes e aproxima-os com o mercado de trabalho, sempre de uma forma nova e atual, contribuindo para a formação do "engenheiro do futuro" e seguindo as expectativas do desenvolvimento do perfil e competências esperados para os novos profissionais.

Um dos aspectos que precisam ser aprimorados no Ruptura UFMG é o envolvimento com estudantes de outras instituições de ensino, o que no formato online se mostrou possível. Acredita-se que a participação de estudantes de outras instituições, além da UFMG, agrega transdisciplinaridade ao evento e uma integração mais ampliada entre os diferentes perfis de estudantes de diferentes instituições. 


\section{Agradecimentos}

O Ruptura UFMG desde a sua primeira edição conta com grandes parceiros, sem os quais não seria possível a sua realização. Agradecimentos devem ser realizados à Joana Bretz, da Optimus, e ao Paulo Victor Guerra, do IEBT.

\section{REFERÊNCIAS}

AGRELA, L. Como formar o engenheiro do Futuro?. Disponível em: https://exame.com/brasil/como-formar-o-engenheiro-do-futuro/. Acesso em: 13 abril. 2021.

BRISCOE, G.; MULLIGAN, C. Digital Innovation: The Hackathon Phenomenon. Creativeworks London Working, n.6, Maio 2014. Disponível em: http://www.creativeworkslondon.org.uk/wp-content/uploads/2013/11/Digital-InnovationThe-Hackathon-Phenomenon1.pdf. Acesso em: 14 abril. 2021.

BUENO, J. R. Mas afinal, o que é empreendedorismo?. Disponível em: https://blog.sebraesc.com.br/o-que-e-empreendedorismo/. Acesso em: 13 abril. 2021.

CIRNE, Stefanie. Impactos da COVID-19 no Setor de Tecnologia e Inovação. Disponível em: $\quad$ https://cadastra.com/pt/insights/impactos-da-covid-19-no-setor-de-tecnologia-einovacao/. Acesso em: 13 abril. 2021.

CONSELHO NACIONAL DE EDUCAÇÃO. Resolução CNE/CSE 02/2019: Institui Diretrizes Curriculares Nacionais do Curso de Graduação em Engenharia. Diário Oficial da União, Brasília, 09 de abril de 2002. 26 de abril de 2019, Seção 1, p. 43-44.

MAGALHÃES, André L. O que é Discord e como criar uma conta para usar no celular e no PC. Disponível em: https://canaltech.com.br/apps/discord-o-que-e-como-criar-conta/. Acesso em: 14 abril. 2021.

MOREIRA, Alessandro F. et al. Práticas Pedagógicas Integradoras e Tecnologias para o Ensino de Engenharia: Programa de Inovação na Educação em Engenharia. In: OLIVEIRA, Vanderli F. et al. DESAFIOS DA EDUCAÇÃO EM ENGENHARIA: Formação em Engenharia, Capacitação Docente, Experiências Metodológicas e Proposições. 1a. ed. Porto Alegre: Ed. Forma Diagramação, 2013, v. 1, p. 215-226.

PAIVA, Carlos E. L. de; ASSAYAG, Elias S.; GARCEZ, Lilyanne R. A importância da participação em Eventos Científicos na formação profissional do engenheiro. In: XLVII Congresso Brasileiro de Educação em Engenharia II Simpósio Internacional de Educação em Engenharia da ABENGE, 2019, Fortaleza. Anais. Manaus, 2019.

PERASSO, V. O que é a $4^{\mathrm{a}}$ revolução industrial - e como ela deve afetar nossas vidas. Disponível em: https://www.bbc.com/portuguese/geral-37658309. Acesso em: 14 abril. 2020.

SILVA, Érica A. da; SILVA, Jacqueline F. da; COSTA, Joana M. L.; ARAÚJO, Carlos W. F. V. de; ALBUQUERQUE, Fábio D. de O. O empreendedorismo e a inovação transformados em modelos de sucesso em uma instituição de ensino superior de Alagoas. In: IV Congresso Brasileiro de Informática na Educação e X Conferência Latino-Americana de Objetos e Tecnologias de Aprendizagem, 2015, Maceió. Anais. Maceió e Recife, 2015. 


\section{RUPTURA UFMG: AN INNOVATION AND ENTREPRENEURSHIP EVENT OF THE SCHOOL OF ENGINEERING OF THE UFMG}

Resumo: This paper presents Ruptura UFMG, an event of innovation and entrepreneurship organized by the ENG200 Program of the School of Engineering of the UFMG in partnership with IEBT and TRIE Engineering. Inspired by the EPICENTRO/Campos do Jordão event and developed along the guidelines of the SCET/UC-Berkeley Entrepreneurship Bootcamp, Ruptura UFMG has student leadership as its milestone. Supported by companies from the Belo Horizonte innovation and entrepreneurship ecosystem, Ruptura UFMG has a disruptive nature, so that the event reinvents itself with each edition, whether held in person or online, promoting a synergy between the industry and the university and providing a favorable environment for exchange of experiences. Fully aligned with the new National Curricular Guidelines for Engineering, Ruptura UFMG is a complementary academic activity in an event format that adds the necessary skills for the formation of the Engineer in this contemporary and globalized world.

Keywords: Innovation and Entrepreneurship; Complementary Academic Activities; Educational Events; Engineering Education Innovation. 